\title{
Multiple Metrics-OLSR in NAN for Advanced Metering Infrastructures
}

\author{
Yakubu Tsado ${ }^{1}$ and Kelum A. A. Gamage ${ }^{1}$ \\ ${ }^{1}$ Department of Engineering \\ Lancaster University \\ Lancaster, UK \\ y.tsado1@1ancaster.ac.uk; k.gamage@lancaster.ac.uk
}

\author{
David Lund $^{2}$, Bamidele Adebisi ${ }^{3}$ \\ ${ }^{2} \mathrm{HW}$ Communications Ltd Lancaster, UK, \\ ${ }^{3}$ Electrical \& Electronics Department, Manchester \\ Metropolitan University, Manchester, UK. \\ d.lund@hwcomms.com; b.adebisi@mmu.ac.uk
}

\begin{abstract}
Routing in Neighbourhood Area Network (NAN) for Smart Grid's Advanced Metering Infrastructure (AMI) raises the need for Quality of Service (QoS)-Aware routing. This is due to the expanded list of applications that will result in the transmission of different types of traffic between NAN devices (i.e smart meters). In wireless mesh network (WMN) routing, a combination of multiple link metrics, though complex, has been identified as a possible solution for QoS routing. These complexities (i.e Np complete problem) can be resolved through the use of Analytical Hierarchy Process (AHP) algorithm and pruning techniques. With the assumption that smart meters transmit IP packets of different sizes at different interval to represent AMI traffic, a case study of the performance of three Optimised Link State Routing (OLSR) link metrics is carried out on a grid topology NAN based WMN in ns-2 network simulator. The best two performing metric were used to show the possibility of combining multiple metrics with OLSR through the AHP algorithm to fulfill the QoS routing requirements of targeted AMI application traffic in NANs.
\end{abstract}

Keywords-AHP algorithm; Link metric; QoS Routing; NAN; AMI.

\section{INTRODUCTION}

Smart Grid (SG) involves a set of sub-systems whose functionalities are crucial and must be established to achieve an intelligent electrical grid system. A typical example of the set of sub-systems is the Advanced Metering Infrastructures (AMI), which integrates a number of devices and technologies to achieve its goals. The infrastructures include smart meters at the consumer end, a two-way communication networks at different infrastructures, data management systems and software application platforms to interface with utilities control end. The smart meters are equipped with advanced electronic meter that collects time-based data for real-time, reliabilityintensive applications and transmit the collected data through available communication networks [1]. Enabling AMI functionalities can be achieved by deploying a static multi-hop wireless mesh network (WMN) that connects a very large number of electric meters to a gateway or data concentrator, which is connected to a control center. However, due to the nature of SG and the challenges of interference and channel errors associated with IEEE 802.11 wireless channels, efficient ad-hoc routing protocols are necessary to extract optimal performance when WMN is deployed in Neighbor Area Network (NAN) for AMI (i.e. smart meters). In WMN, the performance of routing protocol depends significantly on the link metric finding the best paths to a destination. The Qualityof-Service (QoS) requirements for timely and reliable delivery of the varying AMI application traffics in NAN may have different constraints for QoS such as latency, bandwidth and packet delivery. This raises the need for QoS aware routing in smart meters that will be used as mesh nodes in NAN based WMN for AMI.

Adopting suitable mechanisms to enforce different QoS guarantees to network flows depending on AMI application constraints is therefore key to achieving a high level of communication reliability. QoS differentiation in existing communication networks is normally achieved through resource reservation and traffic prioritization. Specifically, various approaches can be employed to prioritize important delay critical data over loss critical data. For instance, many MAC layers (e.g., 802.11e and 802.16) support the specification of different traffic categories and they use scheduling algorithms to provide bandwidth differentiation [2] [3]. However, MAC-based solutions are generally limited to provide QoS guarantees on single communication links. For this reason, there is an increasing awareness that a full-fledged QoS-based architecture as well as QoS routing that allows selecting network routes with sufficient resources for requested QoS parameters are needed to satisfy the different requirements of AMI applications [4]. Existing QoS routing algorithms use link metrics that are tailored towards specific problems, and they lack a simple routing framework that can be easily extended to handle new problems. Most routing algorithms also assume the availability of precise state information about the network, which is impractical in the real world. Furthermore, multiple metrics approaches that have been considered to deal with guaranteeing certain degree of QoS are non-trivial and turn out to be NP-complete ${ }^{1}$. In addressing QoS problems for SG AMI traffics in NAN based WMN, the possibility of using multiple metrics with the Optimised Link State Routing Protocol (OLSR) is explored. This is achieved by evaluating the performance of different link metrics with the OLSR for varying AMI application traffic. Based on the link metric performance, focus is then placed on the proposal of using a heuristic multi-metric approach with OLSR which involve the use of the Analytical Hierarchy Process (AHP)

\footnotetext{
${ }^{1}$ Nondeterministic Polynomial time (NP)-complete problems are problems that are difficult to estimate the number of steps or polynomial time required to solve.
} 
algorithm to support QoS routing and provide optimal path selection for varying smart meter traffic. The idea to use AHP technique for adaptively supporting AMI traffic in NAN is conceived from [5] and [6], which used it for conventional wireless and wired networks. This paper explore the possibility of supporting QoS using this technique on a grid topology NAN based WMN which involve transmission of variable AMI traffics to a data concentrator. The rest of the paper is organized as follows: Section II presents a background on routing for NAN in AMI, Section III explains the reliability improvement process in OLSR protocol for NAN. The performance evaluation of the different OLSR link metric in a WMN NAN scenario is carried out in Section IV, while Section $\mathrm{V}$ presents the Combination of multiple metrics for routing through the AHP algorithm. Finally, Section VI highlights the conclusion.

\section{ROUTING FOR NAN AMI}

A number of routing protocols that have been classified for routing in NAN domain include: Routing Protocol for Low Power and Lossy Networks (RPL) by Winter et al, (2) Geographic routing, (3) Dynamic Source Routing (DSR), (4) Distributed Autonomous Depth-First Routing (DADR), (5) Hybrid Routing Protocol (HYDRO) and (6) Hybrid wireless mesh network protocol (HWMP). There have also been modifications of these protocols to support routing for NANs in the smart grid context. These protocols mostly utilize single metric such as the Extended Transmission Count (ETX) as their link metric. For example, RPL and Hydro use the ETX metric to enable DAG route build tables. Specifically, ETX measures the number of transmissions, including retransmissions, needed to send a unicast packet over a wireless link. For example, if $(i, j)$ are wireless links established between NAN devices $i$ and $j ; P_{i j}$ and $P_{j i}$ signifies the packet loss probability between the wireless link $(i, j)$ in forward and reverse directions respectively. The probability of successful transmission $P s$ between the wireless link $(i, j)$ is therefore computed as $P s_{(i, j)}=\left(\begin{array}{llll}1 & P_{i j}\end{array}\right) \quad\left(\begin{array}{ll}1 & P_{j i}\end{array}\right)$. The expected number of transmissions necessary to deliver the data packet considering both its transmission and successful acknowledgement as required by the IEEE 802.11 protocol can be evaluated as [7]:

$$
\mathrm{ETX}=\frac{1}{P S_{(i, j)}}=\frac{1}{\left(\begin{array}{lll}
1 & P_{i j}
\end{array}\right)\left(\begin{array}{ll}
1 & P_{j i}
\end{array}\right)}
$$

However, ETX is deficient in certain aspects, such as:

- It lacks the ability to model the transmission interference.

- It does not distinguish between links with different capacities, and the loss probability of small probes differs from that of the data packets [7] [8].

As a result, different variations of ETX have been developed with the OLSR routing protocol. The following sub-sections give an insight into the ETX variations with OLSR.

\section{A. ETX Variations in OLSR}

Vast amounts of research have been carried out on link metrics for ad hoc routing protocols. Instead of presenting a survey of the existing literature on routing link metrics, the focus is mainly on research effort related to OLSR routing protocol, which, like other routing protocols, can be implemented as a routing protocol in NAN for AMI. ETX link metric have also been used with OLSR and there exist OLSR's implementation on simulation tools and real network devices, which can be used to develop cross layer metrics for reliable routing in NAN for AMI [7]. As a result of the deficiency in ETX, Other derived ETX metric variations have been proposed, which include:

(1) ETX-ML (Minimum Loss), as the name implies selects paths with minimum loss rates using the probability of successful transmission of forward $d_{f}^{(l)}$ and reverse $d_{r}^{(l)}$ packet delivery ratios, it is depicted in (2).

$$
\text { ETX } M L_{p_{e 2 e}}=\prod_{l \in P_{e 2 e}}\left(d_{f}^{(l)} \times d_{r}^{(l)}\right) \quad 2
$$

Where $\left(d_{f}^{(l)} \times d_{r}^{(l)}\right)$ is the probability of successful delivery of probe packets on link $l$ for end-to-end path $\left(P_{e 2 e}\right)$ from source to destination and from destination to source (reverse direction).

(2) ETX-MD (Minimum Delay), which selects paths between nodes with the lowest sum of transmission delays to the destination.

These metrics trade-off between different performance parameters, the computational burden of some metrics also degrades the performance of the respective protocol [9]. Considering that the functionality of SG is dependent on the ability of different applications meeting certain performance requirements, proposing multiple metric implemented within the existing routing protocols can guarantee the QoS of the variety of traffic in SG AMI network. Though the selection of route based on a combination of multiplicative metric have been proved to be NP-complete [10], techniques such as the AHP and Pruning proposed in [5], have been used to get around the NP-completeness of multiple metric protocols.

\section{B. Potential Problems of OLSR-single metric for NAN AMI}

In the context of deploying single link metric OLSR for NAN in smart grid AMI, a number of new problems can be anticipated. Some of which are:

(1) The simplified routing parameter considered in a single metric cannot consider the many properties and requirements of various AMI applications. However, each of the metric may have significant role in meeting the requirement of a specific application.

(2) The mishandling of latency-tolerant and latency intolerant AMI data. Owing to the different requirements and properties for each AMI application, there is a need to differentiate them in the networking layer.

Thus, measures are needed to mitigate this problem as much as possible to guarantee reliable data transmission in the smart grid network. For example, periodical power quality measuring data has the characteristics of being time tolerant as long as they are successfully delivered. These data can be differentiated from critical data in the network and maintained even in the case of lost links, then transmitted successfully to the destination (utilities or data concentrators) only at the cost 
of higher but tolerable latencies. Different link metrics can also be assigned to varying traffics in the network depending on how efficiently the application traffics are delivered using the metric.

\section{RELIABILITY IMPROVEMENT IN OLSR PROTOCOL FOR NAN}

The proposed reliability improvement of routing for NAN in AMI using OLSR routing protocol is based on two stages. The first stage is to create node architecture for NAN smart meters that consist of a network management perspective at the network layer for routing protocols. The second stage is aimed at using multiple metrics with the OLSR protocol to provide the best available route for targeted AMI application in other to meet their requirements.

\section{A. Multi-metric Node Architecture for NAN devices in AMI}

Link state routing protocols such as OLSR update routing paths to a destination based on metric calculation provided by the link metric being used by OLSR. The selected routes are then used as the default path for data flow to a destination in the network. When variable AMI applications are considered, a combined link metric of ETX, MD and ML with OLSR can measure QoS parameters such as bandwidth, minimum delay and packet losses in order to provide metric evaluation for varying AMI application in NAN.

Fig. 1 presents a structure of the multiple metric-OLSR protocol for NAN in AMI. The node architecture is a modification of HWMP-RE proposed in [11] and CLQM proposed by [12] which defines interfaces between the application and network layer. It is expected to create a platform that will enable evaluation of link parameters and the calculation of paths to NAN destination using multiple link metrics in order to guarantee QoS routing for different targeted application.

\section{B. Module for multi-metric path selection in NAN devices}

An application of the Analytical AHP algorithm for multiple prioritised metrics was proposed for connectionoriented point-to-point communications in [6]. The use of AHP for multiple metrics with OLSR to improve routing in wireless mesh network and deal with high QoS demands was also proposed in [5]. Considering the different applications with special QoS requirement in AMI; changing route priority to meet some special requirements for some traffic is a highly desirable attribute for routing protocols in NANs.

For example, when routing application traffic that are latency intolerant or delay sensitive (DS) as in real time applications, a metric that gives a better end-to-end delay in the network should be considered first priority. In contrast, when routing traffic applications that are latency tolerant or loss sensitive (LS) as in power quality measurement traffic, a metric that has the best delivery reliability performance should be the first priority. The loss sensitive and delay sensitive AMI traffic were represented by power quality traffic transmitting a packet size of 3000 bytes every $3 \mathrm{~s}$ and Wide Area Measurement (WAM) traffic transmitting a packet size of 48 bytes every 0.1 $\mathrm{s}$ respectively [11].

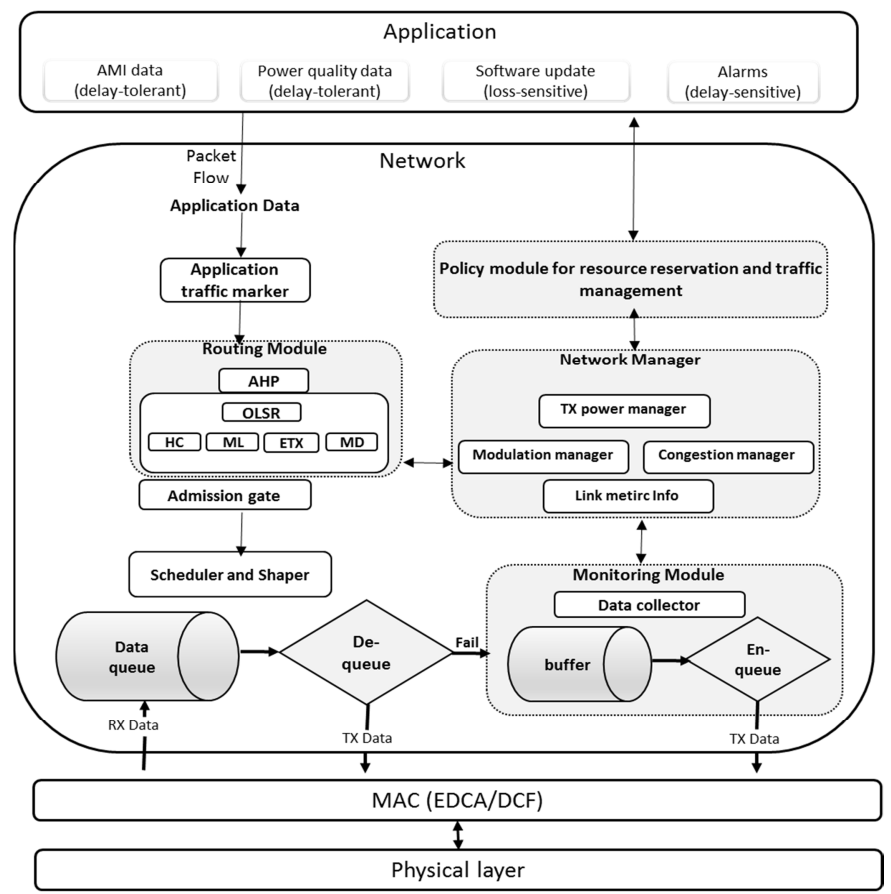

Fig. 1. Structure of the node architecture for implementing multiple-metrics OLSR on NAN devices in AMI

\section{LinK Metric Performance Evaluation (CASE StUdy)}

In this section, the scenario used in carrying out simulations in order to measure the performance of each routing metric is presented. The performance of each metric will also influence the selection of the routing metric for the AHP algorithm.

\section{A. Simulation Scenario}

Simulations were carried out using IPv4 and UDP protocol and the shadowing propagation model in ns-2; which assumes that the average received signal power decreases logarithmically with distance. The simulation topology is represented such that packets are sent towards a destination or data concentrator in a grid topology NAN based WMN. The major difference in simulation parameters in [5] are the topology and the antenna transmission range, which was set such that nodes can only communicate with their closest neighbor in order to show performance of the routing protocol across multiple hops to the destination. The aim of the simulation is to study the performance of OLSR routing link metric on delay and loss sensitive smart metering application. The simulation set up is a representation of smart meters sending traffics for different AMI application to the data concentrator simultaneously in order to demonstrate a worstcase scenario of smart meters communicating with the data concentrator in a NAN.

\section{B. Used Link Metric}

Simulations were carried out to determine the performance of OLSR-ETX, OLSR-ML and OLSR-MD on a smart meter mesh network of grid size 2 by 2 (4) to 7 by 7 (49). The simulations were carried out on ns-2.34 using different seeds for the random number generator. Each simulation was run for 50 seconds and repeated 5 times. A delay sensitive or time critical application in smart grid are often referred to as small 
sized packets which need to be delivered to the destination within a stringent latency (tens of milli seconds), while loss sensitive application traffic are those that must be delivered to the destination regardless of the time it is delivered. Two CBR traffic types were considered: 1) constant bit rate (CBR) flow of 48 bytes transmitting every 0.1 seconds (this could be a WAM traffic, or emergency alarm traffic), and 2) a CBR flow of 3000 bytes every 3 seconds (power quality data). In the simulation, smart meters were required to simultaneously send data to the data concentrator for each of the CBR traffic on ETX, MD and ML link metrics in OLSR so as to choose the best performing metric on each simulation scenario.

\section{Results}

Performance evaluation was measured by estimating the average end-to-end delay, Packet Delivery Ratio (PDR) and throughput on an increasing grid mesh network size. OLSRETX had the highest PDR performance as seen in Fig. 2, but this is not the case with delay. As observed in Fig. 3, OLSRML has the best average delay performance amongst all the other metrics, but, for delay sensitive applications, the delay still exceeds the 200 milli seconds from the 5 by 5 grid size. The frequency at which the critical data is being sent results in queues and loss packets at intermediate or multi-hoping mesh nodes which can contribute to high delays experienced. In real smart metering conditions, some delay critical applications may only be event triggered, for example, fire alarm or security alarm. However, simulating high frequency of delay sensitive application traffic was done to measure the performance of each metric at worst-case scenario. Throughput for both CBR flows on Fig. 4 show that ETX performs better followed by ML and then MD, though it is observed that there is a decline on the average throughput from the 5 by 5 grid size. Since the main goal of the simulation is to select the routing metrics with the overall best performance. Results from the simulation scenarios showed that ETX and ML performed better, and thus should be used with OLSR to improve its routing table computations. Only one CBR flow was considered in each simulation and at 3 by 3 grid mesh size the performance of ETX, MD and ML on both loss and delay sensitive simulation scenarios were above $90 \%$ for PDR and less than 100 milliseconds delay.

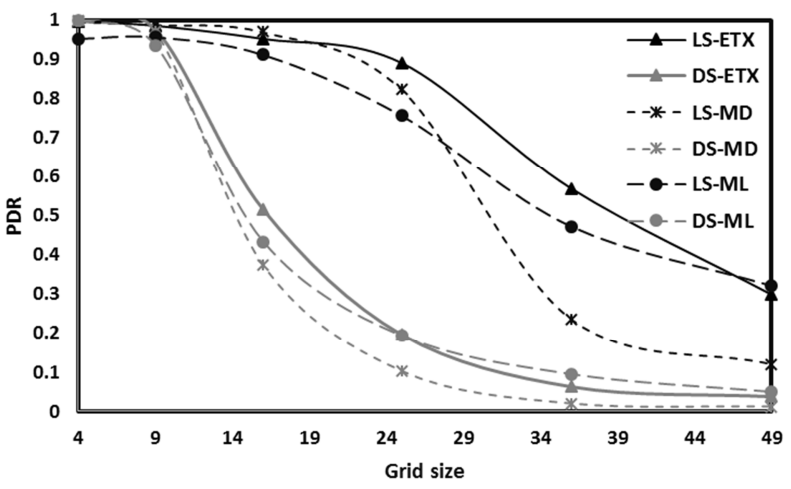

Fig. 2. PDR for delay and Loss sensitive AMI applications

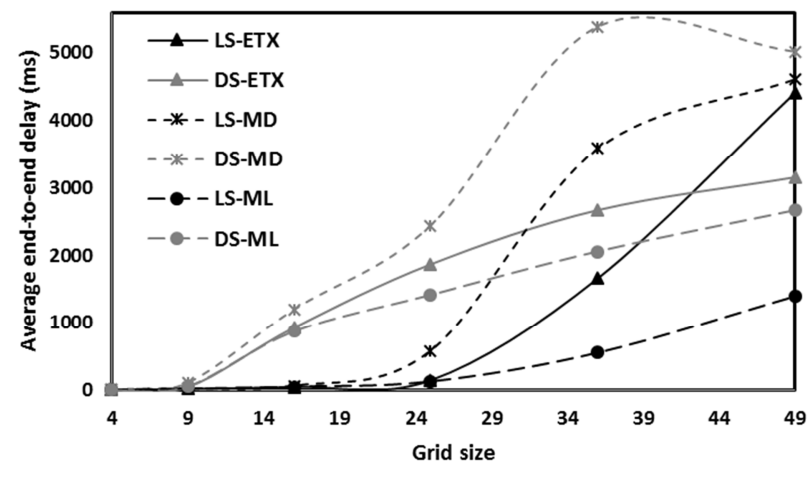

Fig. 3. End-to-end delay for loss and delay sensitive AMI applications

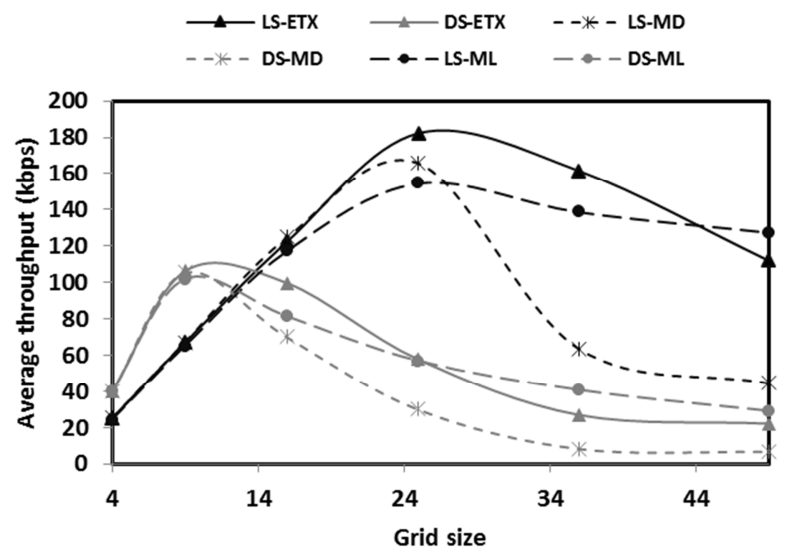

Fig. 4 Average Throughput for delay and Loss sensitive AMI applications

In the next simulation, the number of AMI application traffic sent from each smart meter is incremented from 1 to 4 types of application traffic, in order to evaluate performance of each link metric while sending multiple application traffic flow from the smart meters. Other applications considered are: AMI data applications transmitting 123 bytes of data every 15 seconds and software updates traffic [13]. Results in Fig. 5 and Fig. 6 show a drop in PDR and an increase in average delay on all the link metrics as the number of traffic sent from each meter is increased. ETX and ML still performed better.

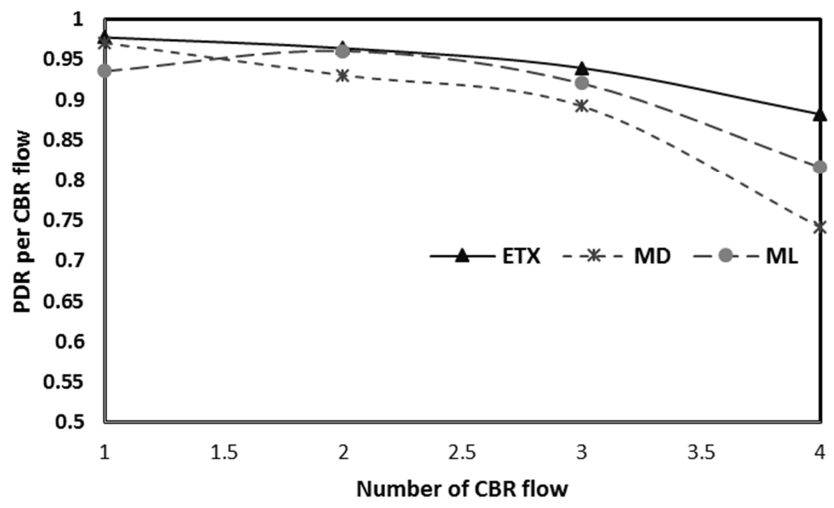

Fig. 5. PDR obtained from multiple traffic transmitted from NAN devices 


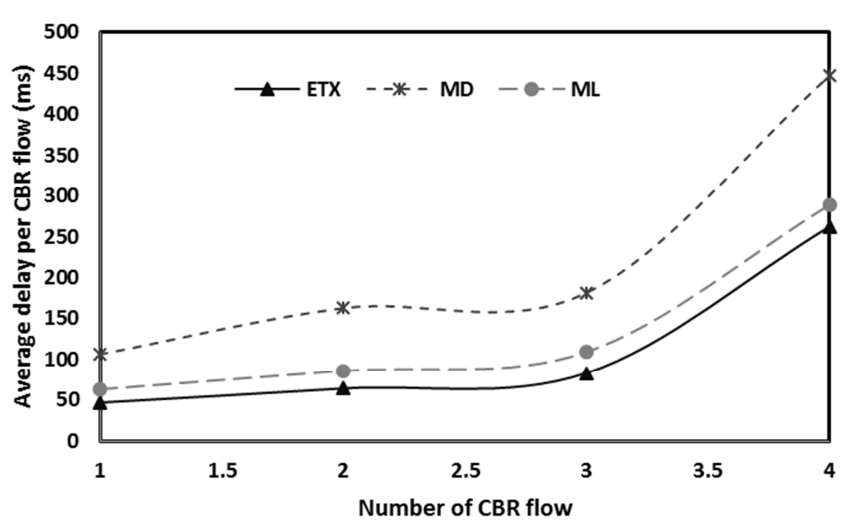

Fig. 6 Average delay obtained from multiple traffic transmitted from NAN devices

\section{COMBINING MULTIPLE LINK METRIC WITH OLSR}

AHP is a robust and flexible multi-criteria decision analysis methodology of measurement developed by [14], it is widely known in the field of decision-making, when different qualitative and/or quantitative criteria must be applied. This methodology is already been used in a number of applications in the field of telecommunications, petroleum pipeline network, project management and health services. By making minor changes to the original AHP methodology, it was used in [5] to decide the best route for multimedia applications to a given destination. This approach is adapted to provide QoS support for AMI application traffic, which are characterized by multiple Quality of Service (QoS) requirements.

\section{A) Applying AHP in QoS routing for AMI based WMN $s$}

In order to illustrate the approach of applying AHP with OLSR, the transmission between Node 0 and Node 8 in the 3 by 3 NAN grid mesh network topology shown in Fig. 7 is considered. Applying AHP will involve three main parts: 1) metric weightings, 2) priority weighting and 3) total score calculations.

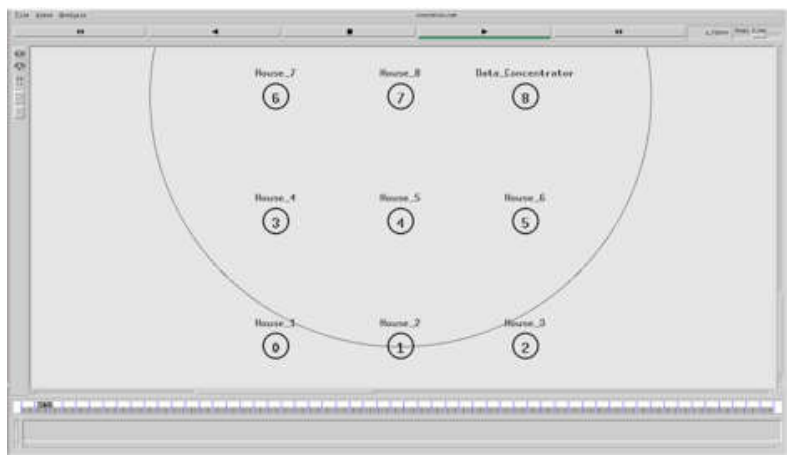

Fig.7. 3 by 3 NAN grid mesh network topology

1) Metric Weighting: Step-A- This step involves finding all possible paths between nodes 0 and 8 . For ease of computation only the four paths between node 0 and 8 were considered. A pruning process will have to be implemented to reduce the number of best paths to be considered especially in larger networks in other to reduce computational complexity and time. Table 1 shows the four paths along with their respective overall values of ETX, and ML.
Table 1 POSSIBLE PATHS FROM NODE 0 - 8

\begin{tabular}{ccccc}
\hline \hline Paths & A & B & C & D \\
\hline \hline Links & 0478 & 058 & 0378 & 0158 \\
& & & & \\
\hline ETX & 7.94 & 10.19 & 5.48 & 7.13 \\
ML & 1.95 & 0.83 & 1.47 & 1.92 \\
& & & & \\
\hline \hline
\end{tabular}

Step-B- Involves the generation of a path-to-path pair-wise comparison matrix (ppcm) for the metrics shown in Table 1. It is used to determine how well a path "scores" on each metric (ETX and ML), by using a pair-wise comparison matrix, with a size of $(N \times N)$, where, $\mathrm{N}$ is the number of paths to the destination. The basic idea of the ppcm is to compare the quantitative metric of ETX and ML of one path with that of all other paths. The matrix calculations are based on the following equation. Where $i$ and $j$ are paths, and $m j$ is the overall value of the metric for path $j$.

- $\quad \operatorname{ppcm}(i, i)=1$, when comparing the same path;

- $\quad \operatorname{ppcm}(j, i)=1 / \operatorname{ppcm}(i, j)$, for reciprocal paths; and

- $\operatorname{ppcm}(I, j)=m j / m i$, for min criterion.

Using the ETX and ML metric presented in Table 1 as an example, the $p p c m$ for ETX is given by:

ppcm $($ ETX $)=\left[\begin{array}{cccc}1 & 1.2834 & 0.6902 & 0.8980 \\ 0.7792 & 1 & 0.5378 & 0.6997 \\ 1.4489 & 1.8595 & 1 & 1.3011 \\ 1.1136 & 1.4292 & 0.7686 & 1\end{array}\right]$

Step-C: The normalized path-path pair-wise comparison matrix (nppcm) is generated by dividing each entry in column $j$ of the ppcm by the sum of the entries in that same column. This results in a normalized matrix in which the sum of the entries in each column is 1 . The formula for calculation is given as follows:

$$
\operatorname{nppcm}(i, j)=\operatorname{ppcm}(i, j) / \sum p p c m \_c o l u m n(j)
$$

The nppcm matrix for ETX is given as:

$$
n p p c m(E T X)=\left[\begin{array}{llll}
0.2303 & 0.2303 & 0.2303 & 0.2303 \\
0.1795 & 0.1795 & 0.1795 & 0.1795 \\
0.3337 & 0.3337 & 0.3337 & 0.3337 \\
0.2565 & 0.2565 & 0.2565 & 0.2565
\end{array}\right]
$$

Step-D: The average normalized path-path pair-wise comparison matrix (anppcm) is calculated for each metric, based on the following equation:

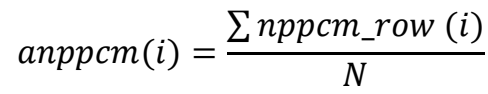

The anppcm matrix is $[n \times N]$ where $\mathrm{n}$ is the number of metrics used ( 2 in this case) and $\mathrm{N}$ is the number of paths. The anppcm for ETX is:

$$
\operatorname{anppcm}(E T X)=\left[\begin{array}{llll}
0.2303 & 0.1795 & 0.3337 & 0.2565
\end{array}\right]
$$

Every metric considered must go through steps B - D to obtain the anppcm for each metric. The complete anppcm for ETX and ML is given as: 
anppcm $=\left[\begin{array}{llll}0.2303 & 0.1795 & 0.3337 & 0.2565 \\ 0.1757 & 0.4128 & 0.2331 & 0.1784\end{array}\right]$

Each line of this matrix refers to the metrics ETX and MD, respectively.

2) Priority weightings: Step-E- This step involves the calculation of the average normalized priority pair-wise comparison matrix (anprpcm) to determine the relative importance of each metric compared with the other metrics. While performing this process in the original AHP, each metric is normally given absolute numbers that reflect the relative importance of one metric compared to the other, based on the decision maker's feelings. Then Steps B - D are performed to find the anprpcm. Modifications proposed by [6] was used, it requires the metrics to be assigned weights in the range of $[0,1]$, where the sum of all the weights equals one as shown in the matrix below:

anprpcm $=[0.5,0.5]$

The required priority of any of the metrics can be set using anprpcm. If the priority is set at 0.5 for both metrics, it means there was no priority considered for any of the metric. In the context of this paper, a priority is set for $\operatorname{ETX}([0.7,0.3])$ and then $\mathrm{ML}([0.3,0.7)]$ in other to view the path choices for different priorities.

3) Total Score Calculation: Step-E- Calculation of the total score for each path can be obtained using the equation below:

Total Normalised score of pat $j \sum_{i=1}^{n}$ (anprpcm $[i]$

Table 2: Path score for different priorities

\begin{tabular}{lcccc}
\hline \hline Paths & $\mathrm{A}$ & $\mathrm{B}$ & $\mathrm{C}$ & $\mathrm{D}$ \\
\hline Total path score & 0.2030 & $\mathbf{0 . 2 9 6 1}$ & 0.2834 & 0.2175 \\
Total score ETX & 0.2139 & 0.2495 & $\mathbf{0 . 3 0 3 5}$ & 0.2331 \\
Total score ML & 0.1921 & $\mathbf{0 . 3 4 2 8}$ & 0.2633 & 0.2019 \\
& & & & \\
\hline \hline
\end{tabular}

Step-F: The path with the maximum total score in Table 3 should be used in the communication between nodes 0 and 8 . As observed in Table 3, path B is the one that has the best link quality when both metrics are given no priority. When ETX is given priority (i.e, anprpcm $=[0.7,0.3]$ ), path $\mathrm{C}$ has the best link quality overall. While, when ML is given priority (i.e, anprpcm $=[0.3,0.7])$, path B has the best quality. This shows that this approach can be very interesting in the implementation of multiple metrics on NAN devices for AMI applications. In order to adaptively guarantee the QoS required for each of the application traffic paths, path $\mathrm{C}$ can be used for loss tolerant traffic, while path $\mathrm{B}$ can be used for delay sensitive traffic.

\section{CONCLUSION}

This paper explores the possibility of using multiple metrics-OLSR to support QoS routing in wireless mesh NANs for AMI through the use of AHP, which have been used in wired and wireless mesh network scenarios. Though the AHP proposed by [6] and [5] concentrated on multimedia application in wired and wireless networks, it does have the potential for routing in NANs for AMI. Two metrics ETX and ML were used along with the AHP algorithm to present a case of adaptively supporting QoS for targeted AMI application in a NAN based WMN. Only four of the best paths to the destination were considered for each link metric in the AHP algorithm, this is rather a simplistic approach as there are more possible best routes to the destination and they increase as the network scales. Considering a large number of possible best paths will of increase computational complexity and time required to select routes. Therefore, good route pruning methods must be developed especially in large networks to reduce the matrix computations and complexities for implementing the OLSR multi-metric algorithm using AHP.

\section{ACKNOWLEDGMENT}

The authors would like to acknowledge the financial support of the ERDF through the Centre for Global EcoInnovation, Lancaster University, UK and the HW Communications Ltd, UK.

\section{REFERENCES}

[1] R. R. Mohassel, A. Fung, F. Mohammadi, and K. Raahemifar, "A survey on advanced metering infrastructure," International Journal of Electrical Power \& Energy Systems, vol. 63, pp. 473-484, 2014.

[2] S.-J. Wu, S.-Y. Huang, and K.-F. Huang, "Efficient Quality of Service scheduling mechanism for WiMAX networks," Computer Communications, vol. 35, pp. 936-951, 2012.

[3] G. Piro, L. A. Grieco, G. Boggia, and P. Camarda, "QoS in wireless LAN: a comparison between feedback-based and earliest due-date approaches," Computer Communications, vol. 35, pp. 298-308, 2012.

[4] D. F. Ramírez and S. Céspedes, "Routing in neighborhood area networks: A survey in the context of AMI communications," Journal of Network and Computer Applications, 2015.

[5] W. Moreira, E. Aguiar, A. Abelém, and M. Stanton, "Using multiple metrics with the optimized link state routing protocol for wireless mesh networks," Simp ${ }^{\wedge}$ Usio Brasileiro de Redes de Computadores e Sistemas DistribuIdos, 2008.

[6] A. M. Alkahtani, M. E. Woodward, and K. Al-Begain, "Prioritised best effort routing with four quality of service metrics applying the concept of the analytic hierarchy process," Computers \& operations research, vol. 33, pp. 559-580, 2006.

[7] S. Paris, C. Nita-Rotaru, F. Martignon, and A. Capone, "Cross-layer metrics for reliable routing in wireless mesh networks," IEEE/ACM Transactions on Networking (TON), vol. 21, pp. 1003-1016, 2013.

[8] J. C. Park, Channel-error and collision aware, secure multihop ad hoc wireless networks: ProQuest, 2008.

[9] N. Javaid, M. Ullah, and K. Djouani, "Identifying design requirements for wireless routing link metrics," in Global Telecommunications Conference (GLOBECOM 2011), 2011 IEEE, 2011, pp. 1-5.

[10] Z. Wang and J. Crowcroft, "Quality-of-service routing for supporting multimedia applications," Selected Areas in Communications, IEEE Journal on, vol. 14, pp. 1228-1234, 1996.

[11] J. Kim, D. Kim, K.-W. Lim, Y.-B. Ko, and S.-Y. Lee, "Improving the reliability of IEEE $802.11 \mathrm{~s}$ based wireless mesh networks for smart grid systems," Journal of Communications and Networks, vol. 14, pp. 629639, 2012.

[12] N. Sarma and S. Nandi, "A Cross-layer QoS Mapping Framework for Mobile Ad Hoc Networks," IETE Technical review, vol. 25, pp. 346358, 2008.

[13] J.-S. Jung, K.-W. Lim, J.-B. Kim, Y.-B. Ko, Y. Kim, and S.-Y. Lee, "Improving IEEE $802.11 \mathrm{~s}$ wireless mesh networks for reliable routing in the smart grid infrastructure," in Communications Workshops (ICC), 2011 IEEE International Conference on, 2011, pp. 1-5.

[14] T. L. Saaty, "The Analytic (Hierarchy) Process, New York, St," Louis ua, 1980 . 